\title{
Solution of Extremely Large Integral-Equation Problems
}

\author{
Ö. Ergül* \\ T. Malas* \\ L. Gürel*†
}

\begin{abstract}
We report the solution of extremely large integral-equation problems involving electromagnetic scattering from conducting bodies. By orchestrating diverse activities, such as the multilevel fast multipole algorithm, iterative methods, preconditioning techniques, and parallelization, we are able to solve scattering problems that are discretized with tens of millions of unknowns. Specifically, we report the solution of a closed geometry containing 42 million unknowns and an open geometry containing 20 million unknowns, which are the largest problems of their classes, to the best of our knowledge.
\end{abstract}

\section{INTRODUCTION}

For the numerical solution of scattering problems in electromagnetics, integral-equation formulations provide accurate results when they are discretized appropriately by using small elements with respect to wavelength. On the other hand, accurate solutions of many real-life problems require discretizations with millions of elements, which result in dense matrix equations with millions of unknowns. For the solution of these large-scale problems involving complicated structures, we employ a parallel implementation of the multilevel fast multipole algorithm (MLFMA) [1].

With the efficient parallelization of MLFMA, problems with tens of millions of unknowns are easily solved on relatively inexpensive computing platforms [2]-[4]. For scattering problems with open surfaces, however, parallel MLFMA is not sufficient to obtain efficient solutions. These problems are inevitably formulated with the electric-field integral equation (EFIE), which usually produces illconditioned matrix equations that are difficult to solve iteratively, especially when the problem size is large [5]. Therefore, we employ advanced preconditioning schemes, such as nested preconditioners based on an approximate MLFMA (AMLFMA) to obtain accelerated convergence even for illconditioned matrix equations obtained from EFIE.

\footnotetext{
*Department of Electrical and Electronics Engineering, Bilkent University, TR-06800, Bilkent, Ankara, Turkey.

$\dagger$ Computational Electromagnetics Research Center (BiLCEM), Bilkent University, TR-06800, Bilkent, Ankara, Turkey.

e-mail: \{ergul,tmalas, lgurel\}@ee.bilkent.edu.tr tel.: +90 3122905750 , fax: +90312 2664192 .
}

\section{PARALLELIZATION OF MLFMA}

MLFMA reduces the complexity of the matrixvector multiplications (MVMs) related to an $N \times N$ dense matrix equation from $O\left(N^{2}\right)$ to $O(N \log N)$. Therefore, it provides the backbone of iterative solution of large scattering problems in electromagnetics. However, it is also desirable to parallelize MLFMA for the solution of many real-life problems that are not easily handled by sequential implementations. On the other hand, parallelization of MLFMA is not trivial due to the complicated structure of the algorithm [6]. Communications between the processors and duplications of the computations reduce the efficiency of the parallelization. In this manner, our implementations involve various parallelization strategies, load-balancing algorithms, optimizations, and many other techniques to improve efficiency without sacrificing accuracy.

\subsection{Near-Field Interactions}

In MLFMA, there are $O(N)$ near-field interactions that are calculated directly and stored in memory. These interactions are distributed among the processors using a load-balancing algorithm. The partitioning of the matrix equation for the nearfield interactions is different from the partitioning for the far-field interactions and the iterative algorithm. Therefore, we perform all-to-one (gather) and one-to-all (scatter) communications in each MVM to match the different partitioning schemes.

\section{$2.2 \quad$ Far-Field Interactions}

In MLFMA, far-field interactions are calculated in a group-by-group manner involving three main stages, i.e., aggregation, translation, and disaggregation, which are performed on a multilevel tree structure constructed by recursively dividing the computational domain into subdomains (clusters). The tree structure is distributed among the processors using a hybrid approach involving different strategies for the lower and higher levels [6]. In the lower (distributed) levels, each cluster is assigned to a single processor. In the higher (shared) levels, however, processor assignments are made on the basis of the fields of the clusters, not on the basis of the clusters themselves. Then, each clus- 
ter is shared by all processors and each processor is assigned to the same portion of the fields of all clusters.

\subsubsection{Aggregation Stage}

During the aggregation stage, radiated fields are calculated at the centers of the clusters from the bottom to the top of the tree structure. Sampling rates for the radiated fields depend on the sizes of the clusters, due to the oscillatory nature of the Helmholtz solutions. Therefore, local interpolation methods are employed to match the different sampling rates of the consecutive levels. In the distributed levels, aggregation stage can be performed at each processor without any communication. Then, an all-to-all communication is required to switch the partitioning strategy from the distributed levels to the shared levels. In the shared levels, aggregation from a level to the next level requires one-to-one communications between the processors, which should be organized carefully. We apply load-balancing algorithms to efficiently distribute the clusters and fields among the processors in the distributed and shared levels, respectively. Finally, the radiation patterns of the basis functions are calculated and stored in memory so that they can be used multiple times during the iterative solution.

\subsubsection{Translation Stage}

In MLFMA, translations are required to compute the interactions between the clusters. For a basis cluster at any level, there are $O(1)$ testing clusters to translate the radiated field into incoming fields. In the distributed levels, some of the translations require one-to-one communications between processors, which are organized carefully by pairing the processors using a communication map. In the shared levels, where each processor is assigned to the same portion of the fields of all clusters, translations are completed without any communication. Using cubic clusters, there are a maximum 316 different translations in each level. The required operators for the translations (or their portions for shared levels) are calculated and stored in memory before the iterative solution. In order to achieve the $O(N)$ complexity for the setup, interpolation algorithms are used during the calculation of the translation operators [7].

\subsubsection{Disaggregation Stage}

During the disaggregation stage, incoming fields at the centers of the clusters are calculated from the

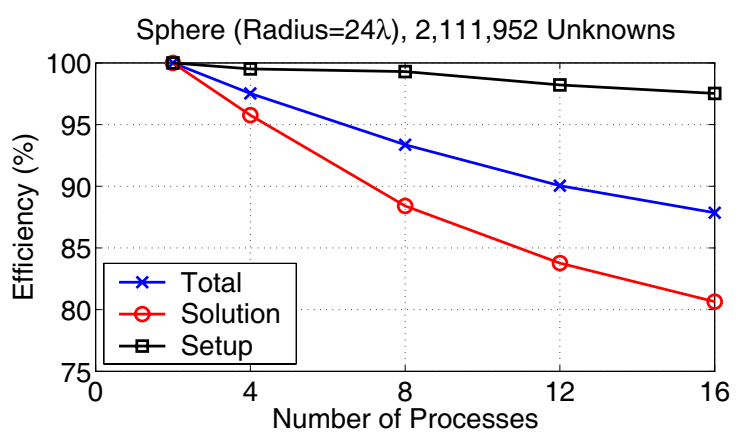

Figure 1: Parallelization efficiency for the solution of a scattering problem involving a sphere of radius $24 \lambda$ discretized with $2,111,952$ unknowns.

top of the tree structure to the lowest level. Transpose interpolations (anterpolations) are employed to match the different sampling rates of the consecutive levels. In the lowest level, incoming fields are multiplied with the receiving patterns of the testing functions and angular integrations are performed to complete the MVM. Similar to the radiation patterns, receiving patterns of the testing functions are also calculated and stored in memory before the iterative solution.

\section{$2.3 \quad$ Efficiency}

To demonstrate the efficiency of the parallelization of MLFMA, we consider a scattering problem involving a sphere of radius $24 \lambda$ discretized with $2,111,952$ unknowns. The problem is solved on a cluster of quad-core Intel Xeon 5355 processors connected via an Infiniband network. Figure 1 presents the parallelization efficiency, when the solution is parallelized into $2,4,8,12$, and 16 processes. Nearfield and far-field interactions are calculated with 2 digits of accuracy. It can be observed that the parallelization of the setup part can be achieved with $97 \%$ efficiency for 16 processes. The solution part, which involves 27 BiCGStab iterations to obtain $10^{-6}$ residual error, is also parallelized efficiently. Due to the communications required during the MVMs, it is difficult to obtain high efficiency for the solution part. However, we are able keep the efficiency above $80 \%$, thanks to effective parallelization strategies and load-balancing algorithms.

\section{SOLUTION OF PROBLEMS INVOLV- ING CLOSED SURFACES}

For the solution of scattering problems involving closed surfaces, we use the combined-field integral equation (CFIE), which is free of the internalresonance problem and produces well-conditioned matrix equations that can be solved efficiently [5]. 
Sphere (Radius=110 $\lambda$ ), 41,883,648 Unknowns

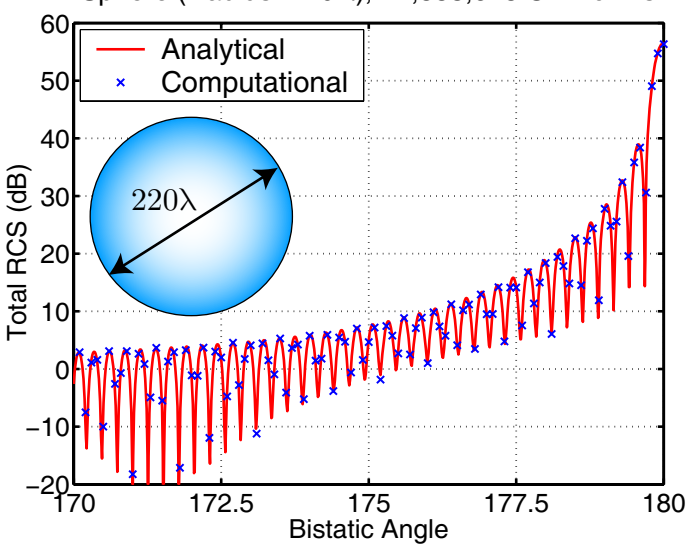

Figure 2: Bistatic RCS (in $\mathrm{dB}$ ) of a sphere of radius $110 \lambda$ discretized with $41,883,648$ unknowns from $170^{\circ}$ to $180^{\circ}$, where $180^{\circ}$ corresponds the forwardscattering direction.

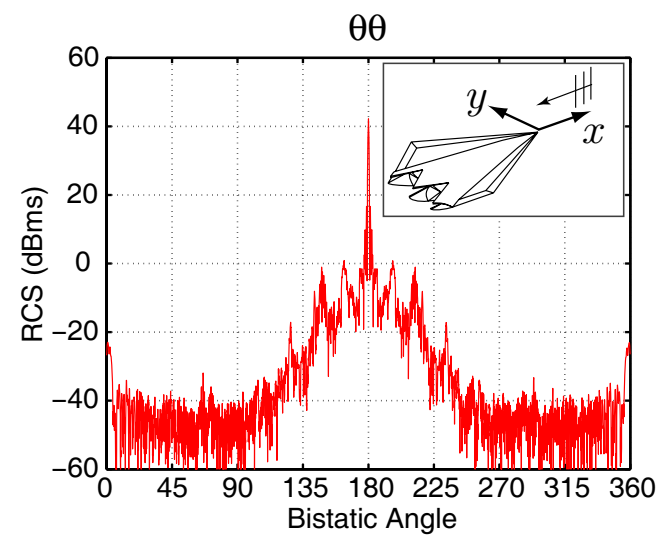

Figure 3: Bistatic RCS (in $\mathrm{dBm}^{2}$ ) of a stealth airborne target Flamme at $16 \mathrm{GHz}$ discretized with $24,782,400$ unknowns.

As an example, we present the solution of a scattering problem involving a sphere of diameter $220 \lambda$ discretized with 41,883,648 unknowns. The solution is parallelized into 16 processes. The MLFMA tree has 3 shared and 6 distributed levels, while the smallest cluster size (in the lowest level) is about $0.21 \lambda$. Both the near-field and far-field interactions are calculated with 2 digits of accuracy. The solution requires only 19 BICGStab iterations to reduce the residual error below $10^{-3}$, when the iterations are accelerated by employing an inexpensive blockdiagonal preconditioner. The setup and solution parts require 274 and 290 minutes, respectively, and the peak memory requirement is 229 GB using the single-precision representation to store the data. Figure 2 depicts the normalized bistatic radar cross section (RCS $/ \lambda^{2}$ ) values in decibels $(\mathrm{dB})$. Analytical values obtained by a Mie-series solution is plotted as a reference from $170^{\circ}$ to $180^{\circ}$, where $180^{\circ}$ corresponds the forward-scattering direction. Fig. 2 shows that the computational values sampled at $0.1^{\circ}$ are in agreement with the analytical solution.

Next, we present the solution of a real-life problem involving the Flamme geometry, which is a stealth airborne target, as detailed in [8]. The maximum dimension of the geometry is 6 meters, which corresponds to $320 \lambda$ at $16 \mathrm{GHz}$. The scattering problem is discretized with $24,782,400$ unknowns using $\lambda / 10$ triangulation. Figure 3 presents the bistatic RCS values in $\mathrm{dBm}^{2}$, when the target is illuminated by a plane wave propagating in the $-x$ direction. The target is on the $x-y$ plane and its nose is directed towards the $x$ axis. Only the $\theta$ polarization is considered and co-polar RCS is plotted as a function of the bistatic angle $\phi$, where $\phi=180^{\circ}$ corresponds to the forward-scattering direction. Solution of the problem is performed by using 10-level MLFMA parallelized into 16 processes. All electromagnetic interactions are calculated with 2 digits of accuracy. The setup and solution parts take 104 and 246 minutes, respectively, while the peak memory requirement is only $139 \mathrm{~GB}$.

\section{SOLUTION OF PROBLEMS INVOLV- ING OPEN SURFACES}

For the solution of large problems involving open surfaces that are formulated by EFIE, strong preconditioners are required to obtain rapid convergence. In MLFMA, near-field interactions that are calculated directly can be used to construct robust preconditioners, such as the sparse approximate inverse (SAI) preconditioner. On the other hand, these preconditioners are not always sufficient for the solution of the dense matrix equations obtained from EFIE, especially when the problem size is large. Therefore, we need stronger preconditioners that provide better approximations to the impedance matrix. For this purpose, we propose AMLFMA, which performs much faster MVMs compared to full MLFMA at the cost of reduced (but controllable) accuracy. We achieve this by carefully decreasing the truncation numbers of the translation operators and the sampling rates of the radiated and incoming fields. AMLFMA is employed in an inner-outer solution scheme [9] and the outer solutions are performed by a flexible solver that allows modifications for the preconditioner. For the inner solutions, MVMs are performed by AMLFMA and the iterative convergence is also accelerated by employing a SAI preconditioner. It is usually sufficient to perform the inner solutions with $10 \%$ residual error. Constructing the 
Patch $(256 \lambda \times 256 \lambda), 20,321,134$ Unknowns

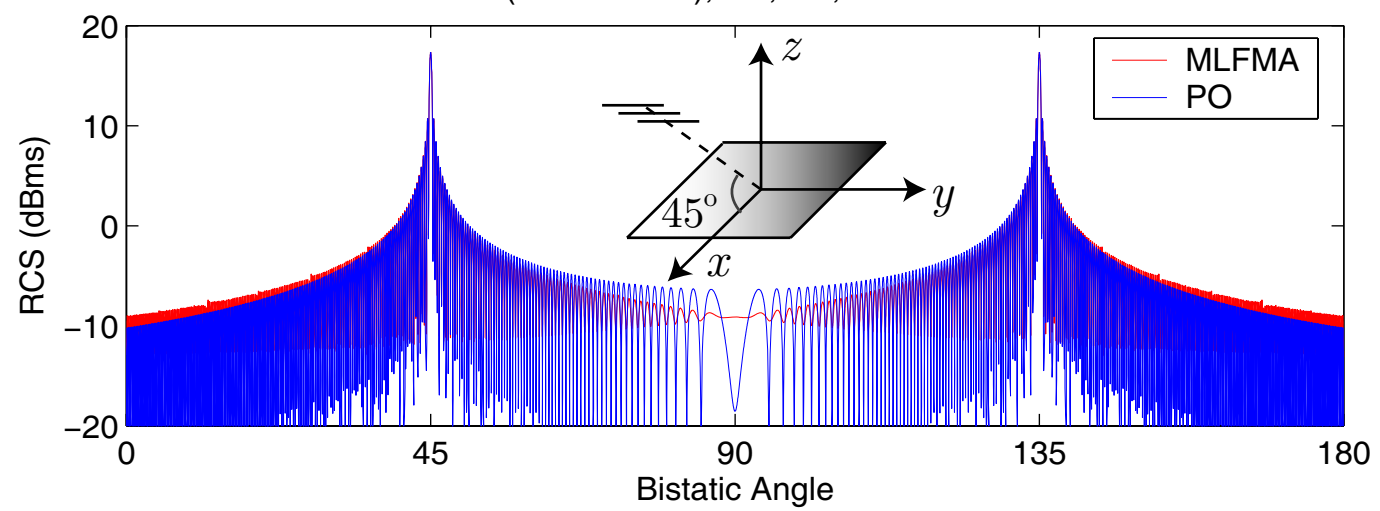

Figure 4: Bistatic RCS (in $\mathrm{dB}$ ) of a $256 \lambda \times 256 \lambda$ patch in the $x$ - $z$ plane from $\theta=0^{\circ}$ to $\theta=180^{\circ}$. The angles $\theta=45^{\circ}$ and $\theta=135^{\circ}$ correspond to specular-reflection and forward-scattering directions, respectively.

AMLFMA carefully by considering the trade-off between the accuracy and efficiency, we are able to solve very large problems involving open surfaces. As an example, Figure 4 presents the co-polar RCS of a $256 \lambda \times 256 \lambda$ square patch illuminated by a plane wave propagating at $45^{\circ}$ from the $z$ axis. Electric field is polarized in the $y$ direction. The scattering problem is discretized with 20,321,134 unknowns and the MLFMA solution is parallelized into 16 processes. The iterative solution, which requires only 9 accurate and 85 approximate MVMs, is completed in 416 minutes. The processing time for the construction of the SAI preconditioner is about 113 minutes. The RCS values are also calculated by employing the physical optics (PO) technique, which is expected to be accurate for the solution of large surfaces. In Figure 4, we observe that PO and MLFMA results agree with each other especially in the specular-reflection and forward-scattering directions, where the $\mathrm{PO}$ results are more reliable.

\section{CONCLUSION}

In this paper, we present the solution of extremely large integral-equation problems involving tens of millions of unknowns. With an efficient parallelization of MLFMA and using robust preconditioning techniques, we are able to solve closed geometries containing 42 million unknowns and open geometries containing 20 million unknowns on relatively inexpensive computing platforms.

\section{Acknowledgments}

This work was supported by the Scientific and Technical Research Council of Turkey (TUBITAK) under Research Grant 105E172, by the Turkish Academy of Sciences in the framework of the Young Scientist Award Program (LG/TUBAGEBIP/2002-1-12), and by contracts from ASELSAN and SSM. Computer time was provided in part by a generous allocation from Intel Corporation.

\section{References}

[1] J. Song, C.-C. Lu and W. C. Chew, "Multilevel fast multipole algorithm for electromagnetic scattering by large complex objects," IEEE Trans. Antennas Propagat., vol. 45, no. 10, pp. 1488-1493, Oct. 1997.

[2] M. L. Hastriter, "A study of MLFMA for large-scale scattering problems," Ph.D. thesis, University of Illinois at Urbana-Champaign, 2003.

[3] G. Sylvand, "Performance of a parallel implementation of the FMM for electromagnetics applications," Int. J. Numer. Meth. Fluids, vol. 43, pp. 865-879, 2003.

[4] L. Gürel and Ö. Ergül, "Fast and accurate solutions of integral-equation formulations discretised with tens of millions of unknowns," Electronics Lett., vol. 43, no. 9, pp. 499-500, Apr. 2007.

[5] L. Gürel and Ö. Ergül, "Extending the applicability of the combined-field integral equation to geometries containing open surfaces," IEEE Antennas Wireless Propagat. Lett., vol. 5, pp. 515-516, 2006.

[6] S. Velamparambil and W. C. Chew, "Analysis and performance of a distributed memory multilevel fast multipole algorithm," IEEE Trans. Antennas Propag., vol. 53, no. 8, pp. 2719-2727, Aug. 2005.

[7] Ö. Ergül and L. Gürel, "Optimal interpolation of translation operator in multilevel fast multipole algorithm," IEEE Trans. Antennas Propagat., vol. 54, no. 12, pp. 3822-3826, Dec. 2006.

[8] L. Gürel, H. Bă̆cl, J. C. Castelli, A. Cheraly, and F. Tardivel, "Validation through comparison: measurement and calculation of the bistatic radar cross section (BRCS) of a stealth target," Radio Sci., vol. 38, no. 3, Jun. 2003.

[9] B. Carpentieri, I. S. Duff, L. Giraud, and G. Sylvand, "Combining fast multipole techniques and an approximate inverse preconditioner for large electromagnetism calculations," SIAM J. Sci. Comput., vol. 27, no. 3, pp. 774-792, 2005. 